\title{
ON A THEOREM OF HARDY AND LITTLEWOOD ON THE POLYDISC ${ }^{1}$
}

\author{
HONG OH KIM \\ Dedicated to Professor Jae Kyu Lim on his sixtieth birthday
}

\begin{abstract}
We prove the polydisc version of the theorem of Hardy and Littlewood on the fractional integral: If $0<\alpha<1 / p$ and if $f \in H^{p}$, then $I^{\alpha} f \in H^{q}$ with $q=\tilde{p} /(1-\alpha p)$ where $I^{\alpha} f$ is the fractional integral of $f$ of order $\alpha$.
\end{abstract}

0. Introduction. If $U$ denotes the open unit disc in the complex plane $\mathbf{C}$, the Hardy space $H^{p}(U), 0<p<\infty$, consists of all functions $f$ holomorphic in $U$ for which

$$
\|f\|_{p}=\sup _{0 \leq r<1} M_{p}(r ; f)<\infty
$$

where

$$
M_{p}(r ; f)=\left(\int_{\theta}^{2 \pi}\left|f\left(r e^{i \theta}\right)\right|^{p} \frac{d \theta}{2 \pi}\right)^{1 / p} .
$$

See [1] for the theory of Hardy spaces. If $f(z)=\sum a_{k} z^{k}$ is holomorphic in $U$, the fractional integral of $f$ of order $\alpha>0$ is defined as

$$
I^{\alpha} f(z)=\sum \frac{\Gamma(k+1)}{\Gamma(k+\alpha+1)} a_{k} z^{k}=\frac{1}{\Gamma(\alpha)} \int_{0}^{1}(1-\rho)^{\alpha-1} f(\rho z) d \rho .
$$

This definition differs from that of Hardy and Littlewood in [4] by a factor $z^{\alpha}$. Flett [2] used $\log (1 / \rho)$ in place of $(1-\rho)$ above for his definition. Since $\log (1 / \rho) \sim 1-\rho$ as $\rho \rightarrow 1$, we see that all these different definitions are "equivalent" for our purpose.

The following theorem was proved originally by Hardy and Littlewood in $[\mathbf{4}, \mathbf{5}]$ and by Flett in [2] with his definition of fractional integral.

THEOREM. If $0<\alpha<1 / p$ and if $f \in H^{p}$, then $I^{\alpha} f \in H^{q}$ with $q=p /(1-\alpha p)$.

A new proof was given to an extended version of this theorem in [7]. The purpose of the present paper is to prove the analogue of this theorem for the polydisc $U^{n}$ of $\mathbf{C}^{n}$. The idea is to iterate the one variable proof of $[\mathbf{7}]$. We only consider the case $n=2$, but the procedure can be repeated for arbitrary $n$. if

A holomorphic function $f$ in $U^{2}$ belongs to the Hardy space $H^{p}\left(U^{2}\right)$ if and only

$$
\|f\|_{p}=\sup _{0 \leq r, s<1} M_{p}(r, s ; f)<\infty,
$$

Received by the editors March 18, 1985 and, in revised form, July 11, 1985.

1980 Mathematics Subject Classification. Primary 30D55, 32A35.

Key words and phrases. Fractional integral, maximal theorem, Hardy space.

1 This research was partly supported by the fund of the Ministry of Education of the Republic of Korea (1984). 
where

$$
M_{p}(r, s ; f)=\left(\int_{0}^{2 \pi} \int_{0}^{2 \pi}\left|f\left(r e^{i \theta}, s e^{i \eta}\right)\right|^{p} \frac{d \theta}{2 \pi} \frac{d \eta}{2 \pi}\right)^{1 / p} .
$$

See [8] for the general theory of Hardy spaces $H^{p}\left(U^{n}\right)$. The fractional integral of $f$ of order $\alpha>0$ is defined as

$$
I^{\alpha, \alpha} f(z, w)=\frac{1}{\Gamma(\alpha)^{2}} \int_{0}^{1} \int_{0}^{1}(1-\rho)^{\alpha-1}(1-\sigma)^{\alpha-1} f(\rho z, \sigma w) d \rho d \sigma .
$$

$f(\cdot, w)$ denotes the function $z \mapsto f(z, w)$ with $w$ fixed and $f_{r, s}$ the function $(z, w) \mapsto$ $f(r z, s w)$. Throughout this paper, $C(\cdots)$ denotes a positive constant depending only on the argument $(\cdots)$ and it may vary from occurrence to occurrence even in the proof of the same theorem.

1. Two more theorems of Hardy and Littlewood on $U^{2}$. For the proof of our main theorem for the case $q \leq 1$, we need two other theorems of Hardy and Littlewood on the polydisc.

1.1. LEMMA. If $f \in H^{p}\left(U^{2}\right)$, then

$$
|f(z, w)| \leq C(p)\|f(z, \cdot)\|_{p}(1-|w|)^{-1 / p}
$$

and

$$
|f(z, w)| \leq C(p)\|f(\cdot, w)\|_{p}(1-|z|)^{-1 / p} .
$$

ProOF. This is a well-known theorem of Hardy and Littlewood [1, Theorem $5.9 ; 4$, Theorem 27] applied to the one variable function $f(z, \cdot)$ with $z$ fixed and $f(\cdot, w)$ with $w$ fixed.

1.2. LEMMA. If $f \in H^{p}\left(U^{2}\right)$, then

$$
\|f(\cdot, w)\|_{p} \leq C(p)\|f\|_{p}(1-|w|)^{-1 / p} .
$$

PROOF.

$$
\begin{aligned}
\|f(\cdot, w)\|_{p}^{p} & =\lim _{r \rightarrow 1} \int_{0}^{2 \pi}\left|f\left(r e^{i \theta}, w\right)\right|^{p} \frac{d \theta}{2 \pi} \\
& \leq C(p)(1-|w|)^{-1} \lim _{r \rightarrow 1} \int_{0}^{2 \pi}\left\|f\left(r e^{i \theta}, \cdot\right)\right\|_{p}^{p} \frac{d \theta}{2 \pi} \quad \text { (Lemma 1.1) } \\
& =C(p)(1-|w|)^{-1} \lim _{r \rightarrow 1} \int_{0}^{2 \pi} \lim _{s \rightarrow 1} \int_{0}^{2 \pi}\left|f\left(r e^{i \theta}, s e^{i \eta}\right)\right|^{p} \frac{d \eta}{2 \pi} \frac{d \theta}{2 \pi} \\
& =C(p)(1-|w|)^{-1} \lim _{\substack{r \rightarrow 1 \\
s \rightarrow 1}} \int_{0}^{2 \pi} \int_{0}^{2 \pi}\left|f\left(r e^{i \theta}, s e^{i \eta}\right)\right|^{p} \frac{d \eta}{2 \pi} \frac{d \theta}{2 \pi} \\
& =C(p)(1-|w|)^{-1}\|f\|_{p}^{p} .
\end{aligned}
$$

Lemma 1.1 and monotone convergence theorem (MCT) are used above.

1.3. LEMMA. If $f \in H^{p}\left(U^{2}\right)$ and if $M(\eta)=\sup _{0 \leq s<1}\left\|f\left(\cdot, s e^{i \eta}\right)\right\|_{p}$, then

$$
\int_{0}^{2 \pi} M(\eta)^{p} \frac{d \eta}{2 \pi} \leq C(p)\|f\|_{p}^{p}
$$


ProOF.

$$
\begin{aligned}
\int_{0}^{2 \pi} M(\eta)^{p} \frac{d \eta}{2 \pi} & =\int_{0}^{2 \pi} \sup _{0 \leq s<1}\left\|f\left(\cdot, s e^{i \eta}\right)\right\|_{p}^{p} \frac{d \eta}{2 \pi} \\
& =\int_{0}^{2 \pi} \sup _{0 \leq s<1} \sup _{0 \leq r<1} \int_{0}^{\pi}\left|f\left(r e^{i \theta}, s e^{i \eta}\right)\right|^{p} \frac{d \theta}{2 \pi} \frac{d \eta}{2 \pi} \\
& \leq \int_{0}^{2 \pi} \int_{0}^{2 \pi} \sup _{0 \leq r, s<1}\left|f\left(r e^{i \theta}, s e^{i \eta}\right)\right|^{p} \frac{d \theta}{2 \pi} \frac{d \eta}{2 \pi} \\
& \leq C(p)\|f\|_{p}^{p} .
\end{aligned}
$$

We applied the complex maximal theorem on the polydisc proved by Zygmund [10] for the last inequality.

A more general version of the following theorem was proved by Frazier [3] and by Jawerth and Torchinsky [6]. For this special case we give still another proof by iterating Ahern's proof $[\mathbf{7}$, Theorem $B]$ of one variable version.

1.4. THEOREM. If $0<p<q<\infty$ and if $f \in H^{p}\left(U^{2}\right)$, then

$$
\int_{0}^{1} \int_{0}^{1}(1-r)^{q / p-2}(1-s)^{q / p-2} M_{1}(r, s ; f)^{q} d r d s<\infty .
$$

PROOF. For the moment, we fix $w$ and set

$$
M(\theta, w)=\sup _{0 \leq r<1}\left|f\left(r e^{i \theta}, w\right)\right| .
$$

By Lemma 1.1 and (1.1), we have

$$
\begin{aligned}
& \int_{0}^{1}(1-r)^{q / p-2}\left|f\left(r e^{i \theta}, w\right)\right|^{q} d r \\
& \quad \leq C(p, q)\|f(\cdot, w)\|_{p}^{q} \int_{0}^{\lambda}(1-r)^{-2} d r+M(\theta, w)^{q} \int_{\lambda}^{1}(1-r)^{q / p-2} d r
\end{aligned}
$$

If $M(\theta, w) \leq\|f(\cdot, w)\|_{p}$, we set $\lambda=0$ in (1.3). (1.2) is then dominated by $C(p, q)\|f(\cdot, w)\|_{p}^{q}$. If $M(\theta, w)>\|f(\cdot, w)\|_{p}$, we set $\lambda=1-\left(\|f(\cdot, w)\|_{p} / M(\theta, w)\right)^{p}$ in (1.3). (1.2) is then dominated by $C(p, q)\|f(\cdot, w)\|_{p}^{q-p} M(\theta, w)^{p}$. Hence, we have, for any $\theta$,

$$
\begin{aligned}
& \int_{0}^{1}(1-r)^{q / p-2}\left|f\left(r e^{i \theta}, w\right)\right|^{q} d r \\
& \quad \leq C(p, q)\left(\|f(\cdot, w)\|_{p}^{q}+\|f(\cdot, w)\|_{p}^{q-p} M(\theta, w)^{p}\right) .
\end{aligned}
$$

We integrate (1.4) with respect to $d \theta / 2 \pi$ and use the complex maximal theorem applied to the one variable function $f(\cdot, w)$ to get

$$
\int_{0}^{1} \int_{0}^{2 \pi}(1-r)^{q / p-2}\left|f\left(r e^{i \theta}, w\right)\right|^{p} \frac{d \theta}{2 \pi} d r \leq C(p, q)\|f(\cdot, w)\|_{p}^{q}
$$

Next, we set

$$
M(\eta)=\sup _{0 \leq s<1}\left\|f\left(\cdot, s e^{i \eta}\right)\right\|_{p}
$$


As we have done above, we have by Lemma 1.2 and (1.6)

$$
\begin{aligned}
& \int_{0}^{1}(1-s)^{q / p-2}\left\|f\left(\cdot, s e^{i \eta}\right)\right\|_{p}^{q} d s \\
& \quad \leq C(p, q)\|f\|_{p}^{q} \int_{0}^{\lambda}(1-s)^{-2} d s+M(\eta)^{q} \int_{\lambda}^{1}(1-s)^{q / p-2} d s .
\end{aligned}
$$

We set $\lambda=0$ if $M(\eta) \leq\|f\|_{p}$ and $\lambda=1-\left(\|f\|_{p} / M(\eta)\right)^{p}$ if $M(\eta)>\|f\|_{p}$ in (1.8). We have then, for any $\eta$,

$$
\int_{0}^{1}(1-s)^{q / p-2}\left\|f\left(\cdot, s e^{i \eta}\right)\right\|_{p}^{q} d s \leq C(p, q)\left(\|f\|_{p}^{q}+\|f\|_{p}^{q-p} M(\eta)^{p}\right) .
$$

We integrate (1.9) with respect to $d \eta / 2 \pi$ and use Lemma 1.3 to get

$$
\int_{0}^{1} \int_{0}^{2 \pi}(1-s)^{q / p-2}\left\|f\left(\cdot, s e^{i \eta}\right)\right\|_{p}^{q} \frac{d \eta}{2 \pi} d s \leq C(p, q)\|f\|_{p}^{q} .
$$

If we combine (1.5) and (1.10), we finish the proof.

The one variable version of the next theorem was proved by Hardy and Littlewood in [4].

1.5. THEOREM. If $0<q \leq 1$ and if $f$ is holomorphic in $U^{2}$, then

$$
\left\|I^{\alpha, \alpha} f\right\|_{q}^{p} \leq C(\alpha, q) \int_{0}^{1} \int_{0}^{1}(1-\rho)^{q \alpha-1}(1-\sigma)^{q \alpha-1} M_{q}^{q}(\rho, \sigma ; f) d \rho d \sigma .
$$

PROOF. If we define

we have

$$
I^{\alpha, 0} f(z, w)=\frac{1}{\Gamma(\alpha)} \int_{0}^{1}(1-\rho)^{\alpha-1} f(\rho z, w) d \rho
$$

$$
I^{\alpha, \alpha} f(z, w)=\frac{1}{\Gamma(\alpha)} \int_{0}^{1}(1-\sigma)^{\alpha-1} I^{\alpha, 0} f(z, \sigma w) d \sigma .
$$

By applying the one variable version of this theorem to $I^{\alpha, 0} f$ with $w$ fixed, we have

$$
\int_{0}^{2 \pi}\left|I^{\alpha, 0} f\left(r e^{i \theta}, w\right)\right|^{q} \frac{d \theta}{2 \pi} \leq C(\alpha, q) \int_{0}^{1}(1-\rho)^{q \alpha-1} \int_{0}^{2 \pi}\left|f\left(\rho r e^{i \theta}, w\right)\right|^{q} \frac{d \theta}{2 \pi} d \rho .
$$

We can also apply the one variable version of this theorem to $I^{\alpha, \alpha} f$ with $z$ fixed because of (1.11) and we get

$$
\int_{0}^{2 \pi}\left|I^{\alpha, \alpha} f\left(z, s e^{i \eta}\right)\right|^{q} \frac{d \eta}{2 \pi} \leq C(\alpha, q) \int_{0}^{1}(1-\sigma)^{q \alpha-1} \int_{0}^{2 \pi}\left|I^{\alpha, 0} f\left(z, \sigma s e^{i \eta}\right)\right|^{q} \frac{d \eta}{2 \pi} d \sigma .
$$

If we combine (1.12) and (1.13) and use Fubini's theorem, we have

$$
\begin{aligned}
\int_{0}^{2 \pi} & \int_{0}^{2 \pi}\left|I^{\alpha, \alpha} f\left(r e^{i \theta}, s e^{i \eta}\right)\right|^{q} \frac{d \theta}{2 \pi} \frac{d \eta}{2 \pi} \\
& \leq C(\alpha, q) \int_{0}^{1} \int_{0}^{1}(1-\rho)^{q \alpha-1}(1-\sigma)^{q \alpha-1} M_{q}^{q}(\rho r, \sigma s ; f) d \rho d \sigma \\
& \leq C(\alpha, q) \int_{0}^{1} \int_{0}^{1}(1-\rho)^{q \alpha-1}(1-\sigma)^{q \alpha-1} M_{q}^{q}(\rho, \sigma ; f) d \rho d \sigma
\end{aligned}
$$

for any $0 \leq r, s<1$. This completes the proof. 


\section{Main theorem.}

2.1. LEMMA. If $f \in H^{p}\left(U^{2}\right)$ and if

$$
M(\eta)=\sup _{0 \leq \sigma<1}\left(\int_{0}^{2 \pi}\left|f\left(r e^{i \theta}, \sigma s e^{i \eta}\right)\right|^{p} \frac{d \theta}{2 \pi}\right)^{1 / p}
$$

then $\int_{0}^{2 \pi} M(\eta)^{p} d \eta / 2 \pi \leq C(p) M_{p}(r, s ; f)^{p}$.

PROOF.

$$
\begin{aligned}
\int_{0}^{2 \pi} M(\eta)^{p} \frac{d \eta}{2 \pi} & =\int_{0}^{2 \pi} \sup _{0 \leq \sigma<1} \int_{0}^{2 \pi}\left|f\left(r e^{i \theta}, \sigma s e^{i \eta}\right)\right|^{p} \frac{d \theta}{2 \pi} \frac{d \eta}{2 \pi} \\
& \leq \int_{0}^{2 \pi} \int_{0}^{2 \pi} \sup _{0 \leq \sigma<1}\left|f\left(r e^{i \theta}, \sigma s e^{i \eta}\right)\right|^{p} \frac{d \theta}{2 \pi} \frac{d \eta}{2 \pi} \\
& =\int_{0}^{2 \pi}\left(\int_{0}^{2 \pi} \sup _{0 \leq \sigma<1}\left|f\left(r e^{i \theta}, \sigma s e^{i \eta}\right)\right|^{p} \frac{d \eta}{2 \pi}\right) \frac{d \theta}{2 \pi} \quad \text { (Fubini) } \\
& \leq C(p) \int_{0}^{2 \pi} \int_{0}^{2 \pi}\left|f\left(r e^{i \theta}, s e^{i \eta}\right)\right|^{p} \frac{d \eta}{2 \pi} \frac{d \theta}{2 \pi} \\
& =C(p) M_{p}(r, s ; f)^{p}
\end{aligned}
$$

We applied the complex maximal theorem to the one variable function $f(z, \cdot)$ to get the last inequality.

Now we are ready to prove our main theorem:

2.2. THEOREM. If $0<\alpha<1 / p$ and if $f \in H^{p}\left(U^{2}\right)$, then $I^{\alpha, \alpha} f \in H^{q}\left(U^{2}\right)$ with $q=p /(1-\alpha p)$.

ProOF. The case $q \leq 1$ follows from Theorems 1.4 and 1.5 since $q / p-2=\alpha q-1$. Now, we assume $q \geq 1$ and set

$$
M\left(r e^{i \theta}, \sigma w\right)=\sup _{0 \leq \rho<1}\left|f\left(\rho r e^{i \theta}, \sigma w\right)\right| .
$$

We write $z=r e^{i \theta}$ and $w=s e^{i \eta}$. By Lemma 1.1,

$$
|f(\rho z, \sigma w)| \leq C(p)\left\|f_{r, s}\left(\cdot, \sigma e^{i \eta}\right)\right\|_{p}(1-\rho)^{-1 / p} .
$$

By (2.1) and (2.2), we have, as in the proof of Theorem 1.4,

$$
\begin{aligned}
& \int_{0}^{1}(1-\rho)^{\alpha-1}\left|f\left(\rho r e^{i \theta}, \sigma s e^{i \eta}\right)\right| d \rho \\
& \leq C(p)\left\|f_{r, s}\left(\cdot, \sigma e^{i \eta}\right)\right\|_{p} \int_{0}^{\lambda}(1-\rho)^{\alpha-1 / p-1} d \rho \\
& \quad+M\left(r e^{i \theta}, \sigma w\right) \int_{\lambda}^{1}(1-\rho)^{\alpha-1} d \rho .
\end{aligned}
$$

If $\left\|f_{r, s}\left(\cdot, \sigma e^{i \eta}\right)\right\|_{p} \geq M\left(r e^{i \theta}, \sigma w\right)$, we set $\lambda=0$ in (2.4). (2.3) is then dominated by $C(\alpha)\left\|f_{r, s}\left(\cdot, \sigma e^{i \eta}\right)\right\|_{p}$. If $\left\|f_{r, s}\left(\cdot, \sigma e^{i \eta}\right)\right\|_{p}<M\left(r e^{i \theta}, \sigma w\right)$, we set

$$
\lambda=1-\left(\left\|f_{r, s}\left(\cdot, \sigma e^{i \eta}\right)\right\|_{p} / M\left(r e^{i \theta}, \sigma w\right)\right)^{p} .
$$


(2.3) is then dominated by

$$
C(\alpha, p)\left\|f_{r, s}\left(\cdot, \sigma e^{i \eta}\right)\right\|_{p}^{\alpha p} M\left(r e^{i \theta}, \sigma w\right)^{1-\alpha p} .
$$

Hence, for any $\eta$ we have

$$
\begin{aligned}
& \int_{0}^{1}(1-\rho)^{\alpha-1}\left|f\left(\rho r e^{i \theta}, \sigma s e^{i \eta}\right)\right| d \rho \\
& \leq C(\alpha, p)\left(\left\|f_{r, s}\left(\cdot, \sigma e^{i \eta}\right)\right\|_{p}+\left\|f_{r, s}\left(\cdot, \sigma e^{i \eta}\right)\right\|_{p}^{\alpha p} M\left(r e^{i \theta}, \sigma w\right)^{1-\alpha p}\right) .
\end{aligned}
$$

Integrating (2.5) with respect to $(1-\sigma)^{\alpha-1} d \sigma$, we get

$$
\begin{aligned}
\left|I^{\alpha, \alpha} f(z, w)\right| \leq C(\alpha, p) & \left(\int_{0}^{1}(1-\sigma)^{\alpha-1}\left\|f_{r, s}\left(\cdot, \sigma e^{i \eta}\right)\right\|_{p} d \sigma\right. \\
& \left.+\int_{0}^{1} M\left(r e^{i \theta}, \sigma w\right)^{1-\alpha p}\left\|f_{r, s}\left(\cdot, \sigma e^{i \eta}\right)\right\|_{p}^{\alpha p}(1-\sigma)^{\alpha-1} d \sigma\right) .
\end{aligned}
$$

We take the $q$-mean on both sides of (2.6) with respect to $d \theta / 2 \pi$ and use the continuous form of Minkowski's inequality to get

$$
\begin{aligned}
&\left(\int_{0}^{2 \pi}\left|I^{\alpha, \alpha}\left(r e^{i \theta}, s e^{i \eta}\right)\right|^{q} \frac{d \theta}{2 \pi}\right)^{1 / q} \\
& \leq C(\alpha, p)\left(\int_{0}^{1}(1-\sigma)^{\alpha-1}\left\|f_{r, s}\left(\cdot, \sigma e^{i \eta}\right)\right\|_{p} d \sigma\right. \\
&\left.+\int_{0}^{1}\left(\int_{0}^{2 \pi} M\left(r e^{i \theta}, \sigma w\right)^{p} \frac{d \theta}{2 \pi}\right)^{(1-\alpha p) / p}\left\|f_{r, s}\left(\cdot, \sigma e^{i \eta}\right)\right\|_{p}^{\alpha p}(1-\sigma)^{\alpha-1} d \sigma\right) \\
& \leq C(\alpha, p) \int_{0}^{1}(1-\sigma)^{\alpha-1}\left\|f_{r, s}\left(\cdot, \sigma e^{i \eta}\right)\right\|_{p} d \sigma .
\end{aligned}
$$

We use the complex maximal theorem

$$
\int_{0}^{1} M\left(r e^{i \theta}, \sigma s e^{i \eta}\right)^{p} \frac{d \theta}{2 \pi} \leq C(p)\left\|f_{r, s}\left(\cdot, \sigma e^{i \eta}\right)\right\|_{p}^{p}
$$

to get the last inequality in (2.7). Next, we set

$$
M(\eta)=\sup _{0 \leq \sigma<1}\left\|f_{r, s}\left(\cdot, \sigma e^{i \eta}\right)\right\|_{p}
$$

By Lemma 1.2, we have

$$
\left\|f_{r, s}\left(\cdot, \sigma e^{i \eta}\right)\right\|_{p} \leq C(p)\left\|f_{r, s}\right\|_{p}(1-\sigma)^{-1 / p} .
$$

By (2.8) and (2.9), we have as before

$$
\begin{aligned}
& \int_{0}^{1}\left\|f_{r, s}\left(\cdot, \sigma e^{i \eta}\right)\right\|_{p}(1-\sigma)^{\alpha-1} d \sigma \\
& \quad \leq C(p)\left\|f_{r, s}\right\|_{p} \int_{0}^{\lambda}(1-\sigma)^{\alpha-1 / p-1} d \sigma+M(\eta) \int_{\lambda}^{1}(1-\sigma)^{\alpha-1} d \sigma .
\end{aligned}
$$


We set $\lambda=0$ if $M(\eta) \leq\left\|f_{r, s}\right\|_{p}$ and $\lambda=1-\left(\left\|f_{r, s}\right\|_{p} / M(\eta)\right)^{p}$ otherwise. We then have for any $\eta$

$$
\begin{aligned}
& \int_{0}^{1}\left\|f_{r, s}\left(\cdot, \sigma e^{i \eta}\right)\right\|_{p}(1-\sigma)^{\alpha-1} d \sigma \\
& \quad \leq C(\alpha, p)\left(\left\|f_{r, s}\right\|_{p}+\left\|f_{r, s}\right\|_{p}^{\alpha p} M(\eta)^{1-\alpha p}\right) .
\end{aligned}
$$

If we take the $q$-mean on both sides with respect to $d \eta / 2 \pi$, we have

$$
\begin{aligned}
& \left(\int_{0}^{2 \pi}\left(\int_{0}^{1}\left\|f_{r, s}\left(\cdot, \sigma e^{i \eta}\right)\right\|_{p}(1-\sigma)^{\alpha-1} d \sigma\right)^{q} \frac{d \eta}{2 \pi}\right)^{1 / q} \\
& \quad \leq C(\alpha, p)\left(\left\|f_{r, s}\right\|_{p}+\left\|f_{r, s}\right\|_{p}^{\alpha p}\left(\int_{0}^{2 \pi} M(\eta)^{p} d \eta\right)^{1 / q}\right) \\
& \leq C(\alpha, p)\left\|f_{r, s}\right\|_{p}
\end{aligned}
$$

by Lemma 2.1. Combining (2.7) and (2.10) we have

$$
M_{q}\left(r, s ; I^{\alpha, \alpha} f\right) \leq C(\alpha, p)\left\|f_{r, s}\right\|_{p}
$$

so $\left\|I^{\alpha, \alpha} f\right\|_{q} \leq C(\alpha, p)\|f\|_{p}$. This completes the proof.

3. Remark. If $f$ is holomorphic in the unit ball $B_{n}$ of $\mathbf{C}^{n}$, we can define the radial fractional integral of $f$ of order $\alpha$ as

$$
I_{r}^{\alpha} f(z)=\frac{1}{\Gamma(\alpha)} \int_{0}^{1}(1-\rho)^{\alpha-1} f(\rho z) d \rho
$$

as in the one variable case. We refer to [8] for the theory of Hardy spaces $H^{p}\left(B_{n}\right)$ of the unit ball $B_{n}$. Since we have the estimate $|f(z)| \leq 2^{n / p}\|f\|_{p}(1-|z|)^{-n / p}$ if $f \in H^{p}\left(B_{n}\right)$ [8, Theorem 7.2.5], and the complex maximal theorem [8, Theorem 5.6.5] for the ball, we can prove as in the one variable case

3.1. THEOREM. If $0<\alpha<n / p$ and if $f \in H^{p}\left(B_{n}\right)$, then $I_{r}^{\alpha} f \in H^{q}$ with $q=n p /(n-\alpha p)$.

\section{REFERENCES}

1. P. L. Duren, Theory of $H^{p}$ spaces, Academic Press, New York, 1970.

2. T. M. Flett, The dual of an inequality of Hardy and Littlewood and some related inequalities, J. Math. Anal. Appl. 38 (1972), 746-765.

3. A. Frazier, The dual space of $H^{p}$ of the polydisc for $0<p<1$, Duke Math. J. 39 (1972), 369-379.

4. G. H. Hardy and J. E. Littlewood, Some properties of fractional integrals. II, Math. Z. 34 (1932), 403-439.

5. _ Theorems concerning mean values of analytic or harmonic functions, Quart. J. Math. 12 (1941), 221-256.

6. B. Jawerth and A. Torchinsky, On a Hardy and Littlewood imbedding theorem, preprint.

7. H. O. Kim. Derivatives of Blaschke products, Pacific J. Math. 114 (1984), 175-191.

8. W. Rudin, Function theory in polydiscs, Benjamin, New York, 1969.

9. _. Function theory in the unit ball of $\mathbf{C}^{n}$, Springer-Verlag, New York, 1980.

10. A. Zygmund, On the boundary value of functions of several complex variables. I, Fund. Math. 36 (1949), 207-235.

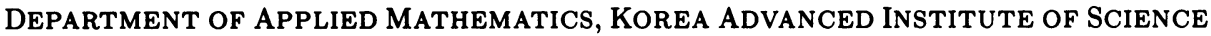
AND TeChNology, P. O. BoX 150, Cheongryangni, SeOul, KoreA 